\title{
Denoising an Image by Denoising its Components in a Moving Frame
}

\author{
Gabriela Ghimpețeanu ${ }^{1}$, Thomas Batard ${ }^{1}$, Marcelo Bertalmío ${ }^{1}$, and Stacey \\ Levine $^{2}$ \\ 1 Universitat Pompeu Fabra, Spain \\ 2 Duquesne University, USA
}

\begin{abstract}
In this paper, we provide a new non-local method for image denoising. The key idea we develop is to denoise the components of the image in a well-chosen moving frame instead of the image itself. We prove the relevance of our approach by showing that the PSNR of a grayscale noisy image is lower than the PSNR of its components. Experiments show that applying the Non Local Means algorithm of Buades et al. [5] on the components provides better results than applying it directly on the image.
\end{abstract}

Keywords: Image denoising, non-local method, differential geometry

\section{Introduction}

Image denoising has been prevalent in the image processing literature for a number of decades. Anisotropic diffusion [14] and total variation based regularization [15] pioneered a rich line of research on edge preserving variational and PDE based methods. More recently, sparsity and self similarity have been used to develop state of the art denoising approaches in the form of patch based and nonlocal methods, e.g. $[5,8,9]$. In fact, it can be argued these techniques are on their way to approaching optimal results $[7,10-12]$.

In [4] the authors demonstrate that for sufficient noise levels, the unit normals of an image have higher PSNR than the image itself, and the curvature of its level lines have even higher PSNR still and thus theoretically should be easier to denoise. This was reflected in the results obtained from smoothing unit normals beginning with [13], as well as smoothing the curvature [4]. In this framework one generates a denoised image whose unit normals, resp. curvature, matches the smoothed results and whose average intensity along level lines matches that of the noisy image. Challenges do still exist however, mainly in developing an optimal reconstruction algorithm, as well as determining mathematically sound approaches for denoising curvature or normal vector field data.

In [1-3], a new approach was developed where one regularizes the components of a noisy image in a moving frame, the results of which are used to reconstruct a denoised image. An immediate benefit of this approach is that the denoised image is obtained from its components in the moving frame using a straightforward 
invertible transform. Furthermore, denoising the components using a variational framework is mathematically sound and experimental results show improvement over comparable approaches.

In this work we demonstrate that patch based methods can also be used to denoise the components of the noisy image in a moving frame, yielding even better results. To this end, the paper is organized as follows. The moving frame approach is described in section 2 . In section 3 we provide experimental evidence that the PSNR of the components in a moving frame is higher than that of the image itself, further justifying this approach. Section 4 contains experimental results demonstrating that patch based methods can be used to denoise the components in this framework, resulting in higher PSNR than comparable approaches. Section 5 contains conclusions and future work.

\section{The moving frame approach}

Let $I$ be a grey-level image defined on a domain $\Omega$ of $\mathbb{R}^{2}$. We construct a surface $S$ embedded in $\left(\mathbb{R}^{3},\|\|_{2}\right)$ parametrized by

$$
\psi:(x, y) \longmapsto(x, y, \mu I(x, y)), \quad \mu>0 .
$$

Therefore, with the standard orthonormal frame $\left(e_{1}, e_{2}, e_{3}\right)$, where $e_{1}=(1,0,0)$, $e_{2}=(0,1,0), e_{3}=(0,0,1)$, the surface $S$ is given by $\psi(x, y)=x e_{1}+y e_{2}+$ $\mu I(x, y) e_{3}$. Note that for $\mu=1$ we obtain the graph of the function $I$.

We construct an orthonormal frame field $\left(Z_{1}, Z_{2}, N\right)$ of $\left(\mathbb{R}^{3},\|\|_{2}\right)$ over $\Omega$, where $Z_{1}, Z_{2} \in \Gamma(T S)$, i.e. $Z_{1}, Z_{2}$ are tangent vector fields of the surface $S$. It follows that $N$ is normal to the surface.

In this paper we choose $Z_{1}$ as the unit vector field indicating the directions of the gradient and $Z_{2}$ as the unit vector field (up to a sign) indicating the directions of the level-lines. Fig. 1 illustrates the construction of the moving frame $\left(Z_{1}, Z_{2}, N\right)$ for a simple image.

Denoting by $P$ the change frame field from $\left(e_{1}, e_{2}, e_{3}\right)$ to $\left(Z_{1}, Z_{2}, N\right)$, we have

$$
P(x, y)=\left(\begin{array}{ccc}
\frac{I_{x}}{\sqrt{\left(I_{x}^{2}+I_{y}^{2}\right)\left(1+\mu^{2}\left(I_{x}^{2}+I_{y}^{2}\right)\right)}} & \frac{-I_{y}}{\sqrt{I_{x}^{2}+I_{y}^{2}}} & \frac{-\mu I_{x}}{\sqrt{1+\mu^{2}\left(I_{x}^{2}+I_{y}^{2}\right)}} \\
\frac{I_{y}}{\sqrt{\left(I_{x}^{2}+I_{y}^{2}\right)\left(1+\mu^{2}\left(I_{x}^{2}+I_{y}^{2}\right)\right)}} & \frac{I_{x}}{\sqrt{I_{x}^{2}+I_{y}^{2}}} & \frac{-\mu I_{y}}{\sqrt{1+\mu^{2}\left(I_{x}^{2}+I_{y}^{2}\right)}} \\
\frac{\mu\left(I_{x}^{2}+I_{y}^{2}\right)}{\sqrt{\left(I_{x}^{2}+I_{y}^{2}\right)\left(1+\mu^{2}\left(I_{x}^{2}+I_{y}^{2}\right)\right)}} & 0 & \frac{1}{\sqrt{1+\mu^{2}\left(I_{x}^{2}+I_{y}^{2}\right)}}
\end{array}\right),
$$

where we have omitted, for clarity purposes, the point coordinates $(x, y)$ where the spatial derivatives $I_{x}$ and $I_{y}$ are evaluated, but it should be noted that when 

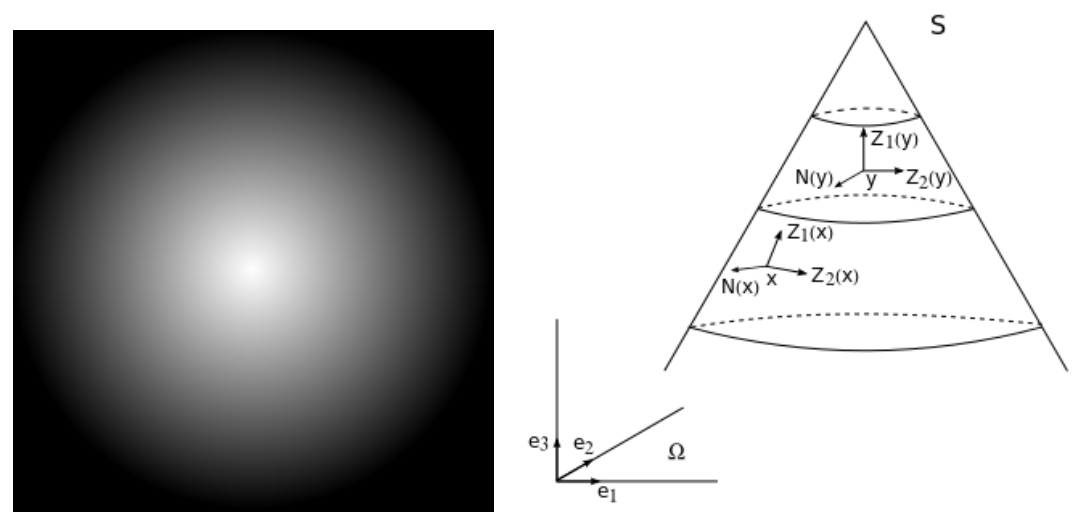

Fig. 1. Orthonormal frame field $\left(Z_{1}, Z_{2}, N\right)$ of $\left(\mathbb{R}^{3},\|\|_{2}\right)$ over $\Omega$. Left: original greylevel image. Right: the frame at two points of the graph $S$ of the image.

we write $I_{x}$ it is implied that we mean $I_{x}(x, y)$, and likewise for $I_{y}$. Note that $Z_{1}, Z_{2}$ are not defined on homogeneous regions of $I$, i.e. at point locations $(x, y)$ where $I_{x}(x, y)=I_{y}(x, y)=0$. In such cases we take $P(x, y)$ as the identity matrix.

Given a grey-level image $I$ and a moving frame associated to $I$ (e.g. the one defined by Eq. (2)), we denote by $J=\left(J^{1}, J^{2}, J^{3}\right)$ the components of $I$ in the new frame, i.e. we have, for each image point $(x, y)$, the following:

$$
\left(\begin{array}{l}
J^{1}(x, y) \\
J^{2}(x, y) \\
J^{3}(x, y)
\end{array}\right)=P^{-1}(x, y)\left(\begin{array}{c}
0 \\
0 \\
I(x, y)
\end{array}\right)
$$

It can easily be shown that the component $J^{2}$ is always zero. Fig. 2 shows the grey-level image "Lena" and its components $J^{1}$ and $J^{3}$ when $\mu=0.05$. We observe that the component $J^{1}$ encodes the gradient information of the image, which was expected since the vector field $Z_{1}$ is pointing in the direction of the gradient. The component $J^{3}$ is similar to the original image, but with highlighted details (contours, textures).

The approach that we take in our proposed framework is that, given a denoising method, it's better to apply it to the components of the noisy image than directly to the image itself. Specifically, let $I_{0}$ be a (noisy) grey-level image and $\left(J_{0}^{1}, J_{0}^{2}, J_{0}^{3}\right)$ its components in a moving frame associated to $I_{0}$ (see formula (3)). The idea developed in [1], [2], [3] is first to apply a regularization method on the components $\left(J_{0}^{1}, J_{0}^{2}, J_{0}^{3}\right)$ instead of the original image $I_{0}$, obtaining regularized components $\left(J^{1}, J^{2}, J^{3}\right)$, and then find the regularized image $I$ whose components are $\left(J^{1}, J^{2}, J^{3}\right)$, through the inverse transform: 


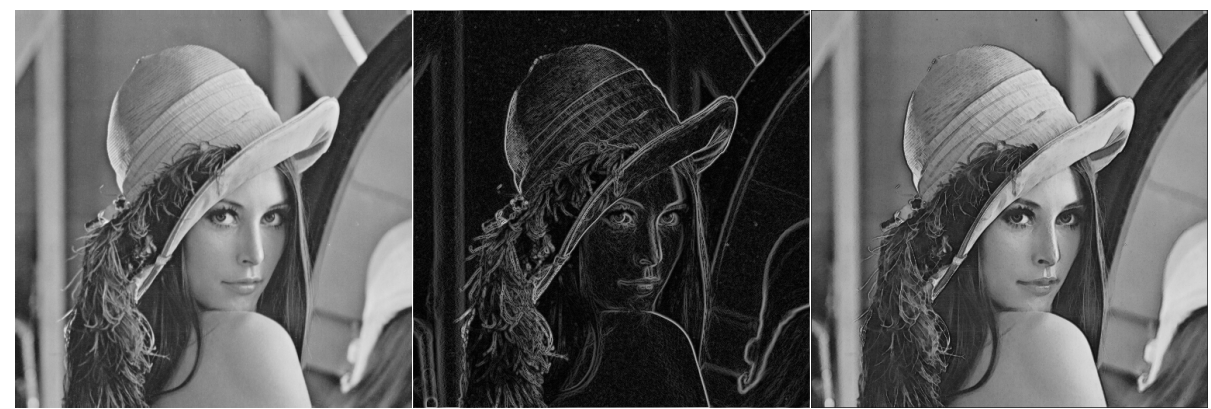

Fig. 2. From left to right: grey-level image "Lena", component $J^{1}$, component $J^{3}$

$$
\left(\begin{array}{l}
\epsilon(x, y) \\
\delta(x, y) \\
I(x, y)
\end{array}\right)=P(x, y)\left(\begin{array}{l}
J^{1}(x, y) \\
J^{2}(x, y) \\
J^{3}(x, y)
\end{array}\right)
$$

The final output of the regularization method is the grey-level image $I$, while $\epsilon$ and $\delta$ should be close to zero everywhere.

In [3], a Euclidean heat diffusion is performed on the function $J_{0}$ yielding an edge-preserving scale-space of the initial image $I_{0}$. In [2], this geometric context was applied to image denoising, dealing with a vectorial extension of the (regularized) Rudin-Osher-Fatemi denoising model. More recently, in [1], the regularized Total Variation in [2] has been replaced by the Vectorial Total Variation (VTV), and this new denoising model is shown to outperform state-of-the-art (local) denoising methods.

\section{The noise level is higher on an image than on its components in a moving frame}

Let $I$ be a clean grey-level image, with components $J^{k}, k=1,2,3$, We add to $I$ Gaussian noise of standard deviation $\sigma$, obtaining a noisy image which we call $I_{0}$. The aim of this section is to show that the components $J_{0}^{k}, k=1,2,3$, of $I_{0}$ are less noisy than $I_{0}$. For this, we compute the PSNR between $J^{k}$ and $J_{0}^{k}$ and see that it is consistently higher than the PSNR between $I$ and $I_{0}$, for different noise levels and for all images in the standard Kodak database (http://rOk.us/graphics/kodak/). The computation of the PSNR requires the specification of the peak value, which is 255 for $I$ and it can be shown that it is also 255 for $J^{3}$, and

$$
\operatorname{peak}\left(J^{1}\right)=255 \times \frac{\sqrt{2} \times 127.5 \mu}{\sqrt{1+2(127.5 \mu)^{2}}}
$$

for $J^{1}$ under the assumption that central differences are used in order to compute the derivatives $I_{x}, I_{y}$. 
Table 1 shows the results for $\sigma=5,10,15,20,25$ and $\mu=1.0,0.1,0.01,0.005$, $0.001,0.0001$. We observe that the PSNR of the components is higher than the PSNR of the image for $\mu \in] 0,0.005]$ and for each noise level aforementioned.

\begin{tabular}{|c|c|c|c|c|c|c|c|}
\hline Noise level & Function & $\mu=1$ & $\mu=0.1$ & $\mu=0.01$ & $\mu=0.005$ & $\mu=0.001$ & $\mu=0.0001$ \\
\hline \multirow{3}{*}{$\sigma=5$} & Component $J^{1}$ & 20.51 & 20.09 & 34.37 & 37.84 & 40.17 & 40.31 \\
\cline { 2 - 8 } & Component $J^{3}$ & 18.56 & 26.02 & 34.24 & 34.22 & 34.19 & 34.19 \\
\cline { 2 - 8 } & Image $I$ & 34.19 & 34.19 & 34.19 & 34.19 & 34.19 & 34.19 \\
\hline \multirow{3}{*}{$\sigma=10$} & Component $J^{1}$ & 19.34 & 15.96 & 28.21 & 31.51 & 33.84 & 33.97 \\
\cline { 2 - 8 } & Component $J^{3}$ & 16.94 & 19.84 & 28.27 & 28.24 & 28.21 & 28.21 \\
\cline { 2 - 8 } & Image $I$ & 28.21 & 28.21 & 28.21 & 28.21 & 28.21 & 28.21 \\
\hline \multirow{5}{*}{$\sigma=20$} & Component $J^{1}$ & 18.32 & 14.16 & 24.44 & 27.79 & 30.09 & 30.22 \\
\cline { 2 - 8 } & Component $J^{3}$ & 16.32 & 16.93 & 24.80 & 24.77 & 24.73 & 24.73 \\
\cline { 2 - 8 } & Image $I$ & 24.73 & 24.73 & 24.73 & 24.73 & 24.73 & 24.73 \\
\hline \multirow{3}{*}{$\sigma=25$} & Component $J^{1}$ & 17.37 & 13.10 & 21.86 & 25.12 & 27.38 & 27.51 \\
\cline { 2 - 8 } & Component $J^{3}$ & 15.98 & 15.22 & 22.38 & 22.33 & 22.28 & 22.27 \\
\cline { 2 - 8 } & Image $I$ & 22.27 & 22.27 & 22.27 & 22.27 & 22.27 & 22.27 \\
\cline { 2 - 8 } & Component $J^{1}$ & 16.47 & 12.36 & 19.89 & 23.03 & 25.25 & 25.38 \\
\cline { 2 - 8 } & Imagent $I$ & 15.77 & 14.10 & 20.50 & 20.44 & 20.37 & 20.37 \\
\hline
\end{tabular}

Table 1. Average values of the PSNR for the components $J^{1}, J^{3}$ and the image $I$ over the Kodak database for different noise levels and values of the parameter $\mu$.

\section{Experiments and comparisons}

As we mentioned above, our general framework is the following: given a denoising method, its better to apply it to the components of the noisy image than directly to the image itself. This was proved to work for local denoising methods in $[2$, 1], and here we want to show it is also the case for non-local denoising methods. Therefore, in this section we take a clean image $I$ and add Gaussian noise to it to create the noisy image $I_{0}$, and then we use a state of the art non-local denoising method like Non-local Means (NLM) [6] to perform the following experiments:

1. Apply NLM to $I_{0}$, obtaining a denoised image which we call $I_{N L M}$.

2. Compute the components $\left(J_{0}^{1}, J_{0}^{2}, J_{0}^{3}\right)$ of $I_{0}$ and apply NLM to them, obtaining the denoised components $\left(J_{d}^{1}, J_{d}^{2}, J_{d}^{3}\right)$, from which we reconstruct a regularized image $I_{d}$ using Eq. (4).

Knowing the ground truth $I$, we can compute and compare the PSNR values of $I_{N L M}$ and $I_{d}$.

We have done this for several noise levels and for all images in the Kodak database, and the numerical results are shown in Table 2. We can see that our approach is consistently better, for all noise levels. The increase in PSNR that we 
obtain, while modest, is in agreement with the optimality bounds estimated in [7, 11,12]. Table 3 lists the parameter values that we have used for each noise level: apart from $\mu$, defined in Eq. (1), we have $\sigma_{k}, k=1,2,3$, which is the parameter value employed by NLM when denoising $J_{0}^{k}$. These parameters are fixed for the whole image database. We must point out that although theoretically $J^{2}=0$, in practice and due to numerical errors in the estimation of the derivatives $I_{x}$ and $I_{y}, J^{2}$ is not exactly 0 and that's why we denoise it anyway.

Figure 3 shows several example results where we can compare the output of our method with that of applying NLM directly to the noisy image. We can see that, with our approach, homogeneous regions are better restored, while details are preserved as well.

Finally, let us briefly mention that the approach that we have proposed for denoising greyscale images can be extended in a natural way to deal with color images. The moving frame will now be five-dimensional, but otherwise the procedure remains the same: computing the components, denoising them, reconstructing an image from these regularized components. Alternatively, we could just apply the proposed approach to each color channel separately. Figure 4 shows an example result, which again compares favorably with NLM applied directly to the image.

\begin{tabular}{|c|c|c|c|c|c|}
\hline PSNR & $\sigma=5$ & $\sigma=10$ & $\sigma=15$ & $\sigma=20$ & $\sigma=25$ \\
\hline$I_{d}$ & 37.48 & 33.59 & 31.57 & 30.12 & 29.00 \\
\hline$I_{N L M}$ & 37.41 & 33.38 & 31.05 & 30.04 & 28.91 \\
\hline
\end{tabular}

Table 2. Average, over the Kodak database, of the PSNR values for $I_{N L M}$ (obtained by applying NLM to the noisy image) and $I_{d}$ (obtained by applying NLM to the components of the noisy image), for different noise levels.

\begin{tabular}{|c|c|c|c|c|c|}
\hline Parameters & $\sigma=5$ & $\sigma=10$ & $\sigma=15$ & $\sigma=20$ & $\sigma=25$ \\
\hline$\mu$ & 0.001 & 0.001 & 0.001 & 0.001 & 0.001 \\
\hline$\sigma_{1}, \sigma_{2}, \sigma_{3}$ & $5,5,6$ & $12,10,11$ & $15,15,16$ & $20,20,21$ & $27,25,26$ \\
\hline
\end{tabular}

Table 3. Parameter values for each noise level.

\section{Conclusions and future work}

In this paper we have shown that the components of a noisy image in a moving frame are less noisy than the image itself, and therefore it's more convenient, given a denoising method such as Non-local Means, to use it to denoise the components and then reconstruct from them a denoised image result, rather than applying Non-local Means directly to the image. We are currently working on determining the optimum parameter values for the color case, testing whether or not we can improve the results by using a denoised frame for the reconstruction, and also trying our framework on $\mathrm{BM} 3 \mathrm{D}[8]$, which is a more recent non-local denoising method. 


\section{Acknowledgments}

This work was supported by European Research Council, Starting Grant ref. 306337, and by Spanish grants AACC, ref. TIN2011-15954-E, and Plan Nacional, ref. TIN2012-38112. S. Levine acknowledges partial support by NSF-DMS \#0915219.

\section{References}

1. T. Batard and M. Bertalmío. On covariant derivatives and their applications to image regularization. Preprint available at http://hal.archives-ouvertes.fr/ hal-00941712.

2. T. Batard and M. Bertalmío. Generalized gradient on vector bundle-application to image denoising. In 4th International Conference on Scale Space and Variational Methods in Computer Vision (A. Kuijper et al. Eds.), LNCS 7893, pages 12-23. Springer, 2013.

3. T. Batard and M. Berthier. Spinor Fourier transform for image processing. IEEE Journal of Selected Topics in Signal Processing (Special Issue on Differential Geometry in Signal Processing, 7(4):605-613, 2013.

4. M. Bertalmío and S. Levine. Denoising an Image by Denoising Its Curvature Image. SIAM J. Imaging Sci., 7(1):187-211, 2014.

5. A. Buades, B. Coll, and J-M. Morel. A non-local algorithm for image denoising. In Computer Vision and Pattern Recognition, 2005. CVPR 2005. IEEE Computer Society Conference on, volume 2, pages 60-65. Ieee, 2005.

6. A. Buades, B. Coll, and J-M. Morel. Non-local Means Denoising. Image Processing On Line, 2011.

7. P. Chatterjee and $\mathrm{P}$ Milanfar. Is denoising dead? IEEE Transactions on Image Processing, 19(4):895-911, 2010.

8. K. Dabov, A. Foi, V. Katkovnik, and K. Egiazarian. Image denoising by sparse 3$\mathrm{d}$ transform-domain collaborative filtering. Image Processing, IEEE Transactions on, 16(8):2080-2095, 2007.

9. M. Elad and M. Aharon. Image denoising via sparse and redundant representations over learned dictionaries. IEEE Trans. on Image Processing, 15(12):3736-3745, 2006.

10. M. Lebrun, M. Colom, A. Buades, and J. M. Morel. Secrets of image denoising cuisine. Acta Numer., 21:475-576, 2012.

11. A. Levin and B. Nadler. Natural image denoising: Optimality and inherent bounds. In Computer Vision and Pattern Recognition (CVPR), 2011 IEEE Conference on, pages 2833-2840. IEEE, 2011.

12. A. Levin, B. Nadler, F. Durand, and W.T. Freeman. Patch complexity, finite pixel correlations and optimal denoising. Technical report, MIT - Computer Science and Artificial Intelligence Laboratory, 2012.

13. M. Lysaker, S. Osher, and X.C. Tai. Noise removal using smoothed normals and surface fitting. Image Processing, IEEE Transactions on, 13(10):1345-1357, 2004.

14. P. Perona and J. Malik. Scale-space and edge detection using anisotropic diffusion. Pattern Analysis and Machine Intelligence, IEEE Transactions on, 12(7):629-639, 1990.

15. L.I. Rudin, S. Osher, and E. Fatemi. Nonlinear total variation based noise removal algorithms. Physica D: Nonlinear Phenomena, 60(1-4):259-268, 1992. 


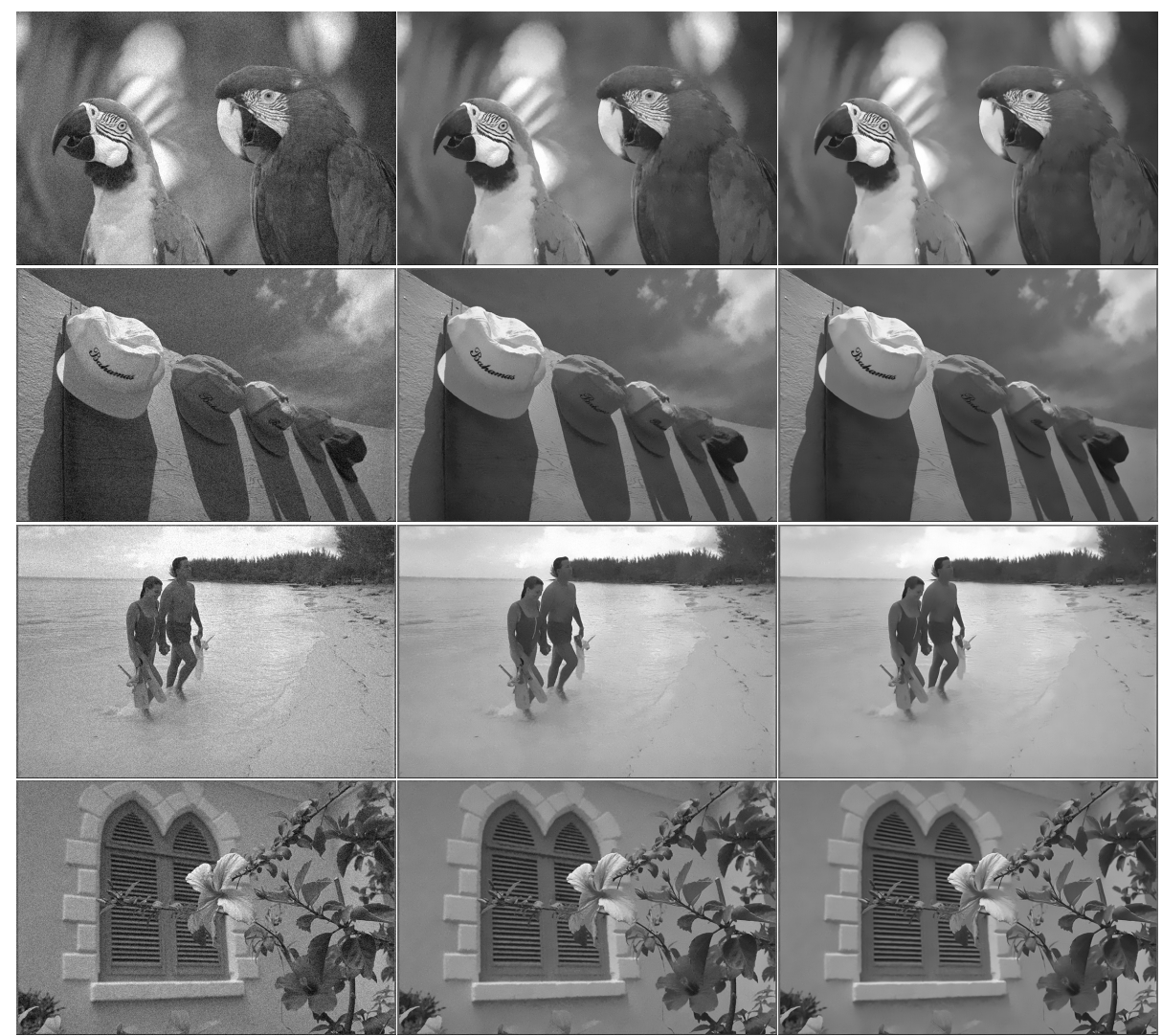

Fig. 3. Comparing the output of our method with that of applying NLM directly to the noisy image. Left: original noisy image. Middle: NLM result. Right: our result. First row: NLM PSNR $=33.41$, our result $\mathrm{PSNR}=34.88$. Second row: $\mathrm{NLM} \mathrm{PSNR}=33.10$, our result $\mathrm{PSNR}=34.18$. Third row: $\mathrm{NLM} P S N R=32.55$, our result $\mathrm{PSNR}=33.23$. Fourth row: $\mathrm{NLM} P S N R=32.24$, our result $\mathrm{PSNR}=33.43$.

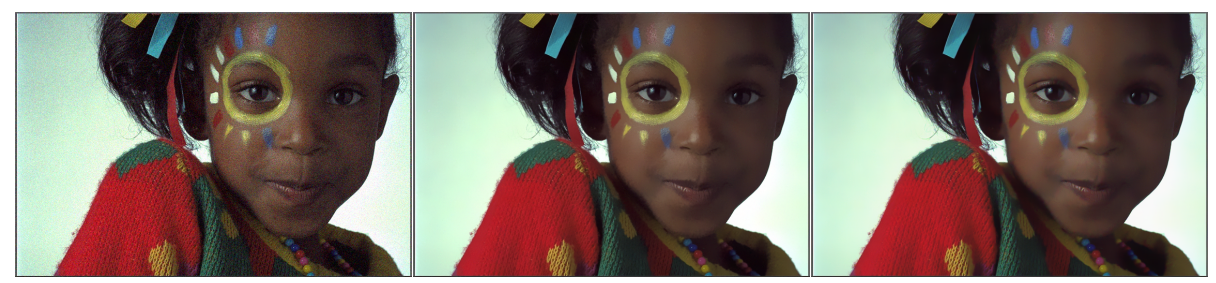

Fig. 4. Comparing the output of our method with that of applying NLM directly to the noisy image. Left: original noisy image. Middle: NLM result. Right: our result. 